\title{
Minimally invasive mitral valve repair using external clamping- pearls and pitfalls
}

\author{
Karel M. Van Praet ${ }^{1,2}$, Markus Kofler ${ }^{1}$, Matteo Montagner ${ }^{1}$, Roland Heck ${ }^{1}$, Dirk Eggert-Doktor ${ }^{3}$, Christof \\ Stamm $^{1,2}$, Axel Unbehaun ${ }^{1}$, Stephan Jacobs ${ }^{1}$, Volkmar Falk ${ }^{1,2,4,6}$, Jörg Kempfert ${ }^{1,2}$ \\ ${ }^{1}$ Department of Cardiothoracic and Vascular Surgery, German Heart Center Berlin, Berlin, Germany; ${ }^{2}$ DZHK (German Center for Cardiovascular \\ Research), Partner Site Berlin, Berlin, Germany; ${ }^{3}$ Institute for Anesthesiology, German Heart Center Berlin, Berlin, Germany; ${ }^{4}$ Department of \\ Cardiovascular Surgery, Charité - Universitätsmedizin Berlin, Berlin, Germany; ${ }^{5}$ Berlin Institute of Health (BIH), Berlin, Germany; ${ }^{6}$ Department of \\ Health Sciences, ETH Zürich, Translational Cardiovascular Technologies, Zürich, Switzerland \\ Contributions: (I) Conception and design: All authors; (II) Administrative support: All authors; (III) Provision of study materials or patients: All \\ authors; (IV) Collection and assembly of data: All authors; (V) Data analysis and interpretation: All authors; (VI) Manuscript writing: All authors; (VII) \\ Final approval of manuscript: All authors. \\ Correspondence to: Karel M. Van Praet, MD. Department of Cardiothoracic and Vascular Surgery, German Heart Center Berlin (Deutsches \\ Herzzentrum Berlin), Augustenburger Platz 1, 13353 Berlin, Germany. Email: vanpraet@dhzb.de.
}

\begin{abstract}
Minimally invasive mitral valve surgery is safe, effective and increasingly popular for patients with valvular pathology. After minimally invasive mitral valve surgery, patients report less pain, shorter hospital stay and better cosmetic results compared to other more invasive approaches. To perform this, cardiopulmonary bypass is needed and aortic occlusion is a critical step in its setup. It is performed worldwide with the use of either trans-thoracic external aortic cross-clamping or endo-aortic balloon occlusion. Both possibilities present peculiar advantages and limitations whose current evidence is based on few observational studies. This article will present the use of the trans-thoracic external aortic cross-clamp in further detail within the setting of minimally invasive mitral valve surgery. We conducted a literature search and discuss the differences in perioperative outcomes and complications between the endo-aortic balloon and the transthoracic external aortic cross-clamp technique during elective minimally invasive mitral valve surgery. According to a systematic review and meta-analysis published in 2019 by the Bristol group, the endo-aortic balloon technique was associated with a higher risk of aortic dissection. Endo-aortic balloon occlusion with aortic cannulation offers the shortest extracorporeal support times. Both techniques are safe and have similar rates of cerebrovascular accident and survival.
\end{abstract}

Keywords: Mitral valve repair; endo-aortic balloon; balloon clamping; aortic clamping; cross-clamping

Received: 29 December 2019; Accepted: 27 May 2020; Published: 05 October 2020.

doi: 10.21037 /jovs-2019-17

View this article at: http://dx.doi.org/10.21037/jovs-2019-17

\section{Background}

Minimally invasive cardiac surgery has increasingly been used for patients with valvular pathology (1-3). There are two techniques used in aortic clamping: trans-thoracic external aortic cross-clamping (TTC) and endo-aortic balloon occlusion (EABO) (4-6) as depicted in Figure 1. Both options present specific advantages and limitations whose current evidence is based on few retrospective observational studies only (7-9). Rival et al. (10) recently published the results of their systematic review and meta-analysis on the topic of TTC versus EABO in minimally invasive mitral valve surgery (MIMVS). They came to the conclusion that both techniques are safe and have similar rates of cerebrovascular accident (CVA) and survival (10). EABO was associated with a higher risk of aortic dissection (10). EABO with aortic cannulation offers the shortest extracorporeal support times (10). In their opinion, there is little evidence to support the adoption of one technique 
A

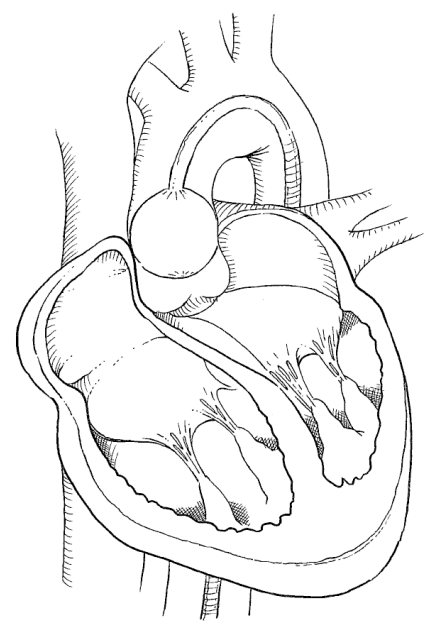

B

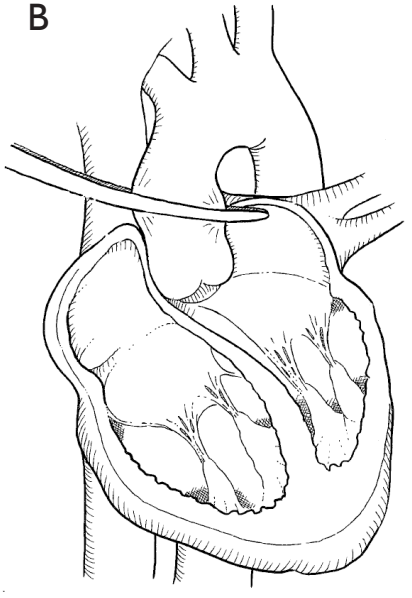

Figure 1 Schematic drawings of aortic cross-clamping techniques. (A) Endoaotic cross-clamping using an endo-clamp (balloon occlusion) (EABO). By using an aortic endoclamp placed through a side-limb of the femoral arterial CPB-cannula, aortic cross-clamping, antegrade cardioplegia administration, and aortic root venting can be accomplished. The endo-clamp is a multi-lumen catheter with an inflatable balloon at its distal end which provides endo-aortic clamping. A central lumen can provide antegrade cardioplegia delivery or alternatively aortic root venting. A second tip lumen allows monitoring of aortic root pressure. (B) Trans-thoracic external aortic cross-clamping (TTC) technique.

over another other than personal choice (10). In such a position of true equipoise, they would therefore encourage the careful design of a randomized controlled trial (RCT) comparing TTC and EABO in specific participant subgroups (10). Our article serves as a step-by-step guide on how to perform MIMVS using the trans-thoracic external aortic cross-clamp and elaborates on the pearls and pitfalls of this technique. Additionally, we performed a literature search with the primary objective to evaluate outcomes and the incidence of major complications associated with these two techniques.

\section{Step-by-step}

Preoperative planning of the procedure utilizing ECG synchronized computed tomographic imaging of the chest and the aorto-ilio-femoral vasculature

The optimal preoperative computed tomographic angiography (CTA) extends from the upper thoracic aperture to the lesser trochanter. This is to include the thoracic cage as well as the thoracic and abdominal aorta, the iliac arteries and common femoral arteries, the latter constituting the most common vascular access site in MIMVS. When assessing the chest CT-scan, there are several factors that need to be taken into consideration: the elimination of ascending aortic disease, the detection of significant mitral valve (MV) annular calcification and the evaluation of other anatomic abnormalities. Ascending aortic disease must be ruled out because the aortic occlusion is accomplished with either an external crossclamp or by means of EABO. In aortas with a diameter of more than $4 \mathrm{~cm}$, complete occlusion with an endoaortic balloon becomes less consistent. For inexperienced surgeons, significant MV annular calcification is a relative contraindication for MIMVR. The fourth intercostal space is most commonly used for the working port; however, a preoperative CT-scan may help guide the surgeon above or below this space to be better aligned with the mitral valve plane. With respect to anatomic abnormalities, they should be assessed because they could potentially complicate any surgery. These abnormalities include damage to the chest wall, ribs or diaphragm. MIMVR can be performed with central cannulation techniques (11) although to minimize the chest incision and rib spreading and to provide clear access to the MV, most surgeons prefer peripheral cannulation. Thorough knowledge of peripheral vascular anatomy is needed for this approach and a CTA of the chest, abdomen and pelvis, preferably with contrast, supplies the most useful information. Without 

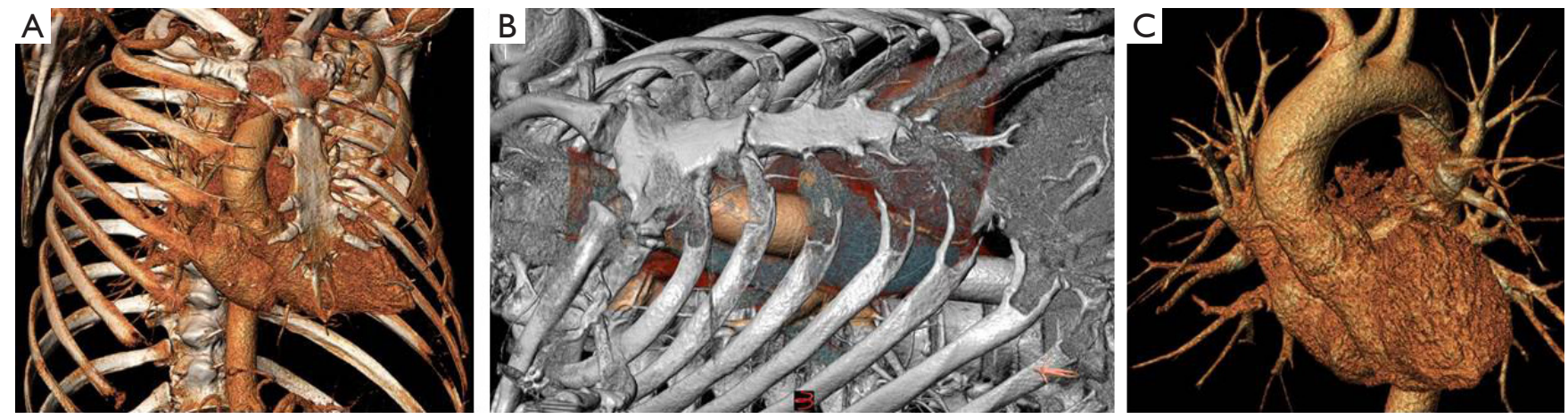

Figure 2 Reconstruction and planning of the main thoracic access site and working port. (A,B) 3D reconstructions of the thoracic cage produced with the 3 mensio Structural Heart software package. The fourth intercostal space will be used as the main working port. (C) 3D reconstruction of the left heart system, the ascending aorta and the aortic arch. The picture does not show any aneurysmal disease, thrombus or calcium.

contrast, the CT-scan is still useful but may not expose subtleties in soft plaque which are important for peripheral cannulation and retrograde arterial perfusion (RAP). When planning femoral arterial access, the femoral and iliac arteries and aorta should have minimal aneurysmal disease, thrombus or calcium. Evidence of an iliac or femoral artery dissection is a contraindication for peripheral arterial cannulation. Patients with a history of peripheral vascular disease should undergo evaluation with lower extremity non-invasive studies and/or lower extremity CTA, as cannulation can result in lower-extremity ischemia while on CPB. Nevertheless, in cases with borderline arterial femoral diameter, the surgeon can still opt for additional distal leg perfusion through an extra smaller cannula. The venous anatomy should also be examined. It is unusual that femoral venous access cannot be used, however patients with a history of deep vein thrombosis, and particularly those with an inferior vena cava (IVC) filter, may require a CT venogram to ensure the IVC is patent. Besides, threedimensional (3D) reconstructions of preoperative CT-scans greatly support the better understanding of complex cardiac anatomy, preoperative surgical planning and improve communication within the multidisciplinary team. They often add important new anatomical findings and prompt alternative operative scenarios. As depicted in Figure $2 A$ and $2 B$, a $3 \mathrm{D}$ reconstruction of a preoperative CTA precisely determines the ideal position for the chest incision. On the other hand, as shown in Figure $2 C$, the ascending thoracic aorta does not show any calcifications nor atherosclerotic plaques meaning that cross-clamping the ascending aorta with an external direct trans-thoracic clamp would be safe.
Additionally, CT-scans can also show significant coronary artery disease. Figure 3 displays the workflow of several modules from the 3 mensio Structural Heart software package for the assessment of the surgical approach route. Of course CT post processing could be performed with alternative software tools such as syngo ${ }$.via osiriX, TeraRekon just to mention a few options commonly used.

\section{Patient positioning \& anaesthetics}

The patient is intubated with a standard single lumen endotracheal tube. The topic of whether or not a double lumen tube is required for this procedure is still a matter of discussion within the surgical community. The patient is positioned supine with a small pillow under the right scapula to elevate the right hemithorax. If trans-thoracic external aortic cross-clamping is planned, placement of one unilateral radial arterial catheter is sufficient in contrast to the EABO technique where bilateral radial pressure monitoring is required.

\section{Surgical access (Video 1)}

A $4 \mathrm{~cm}$ right lateral minithoracotomy, inframammary in men and in the submammary crease in women, is used to enter the thorax through the fourth intercostal space (ICS). A small thoracic soft-tissue retractor is utilized to spread the tissue without the use of a metal rib retractor. An alternative access as a variation of the standard right lateral minithoracotomy is the periareolar approach. This minimally invasive periareolar approach for surgical 

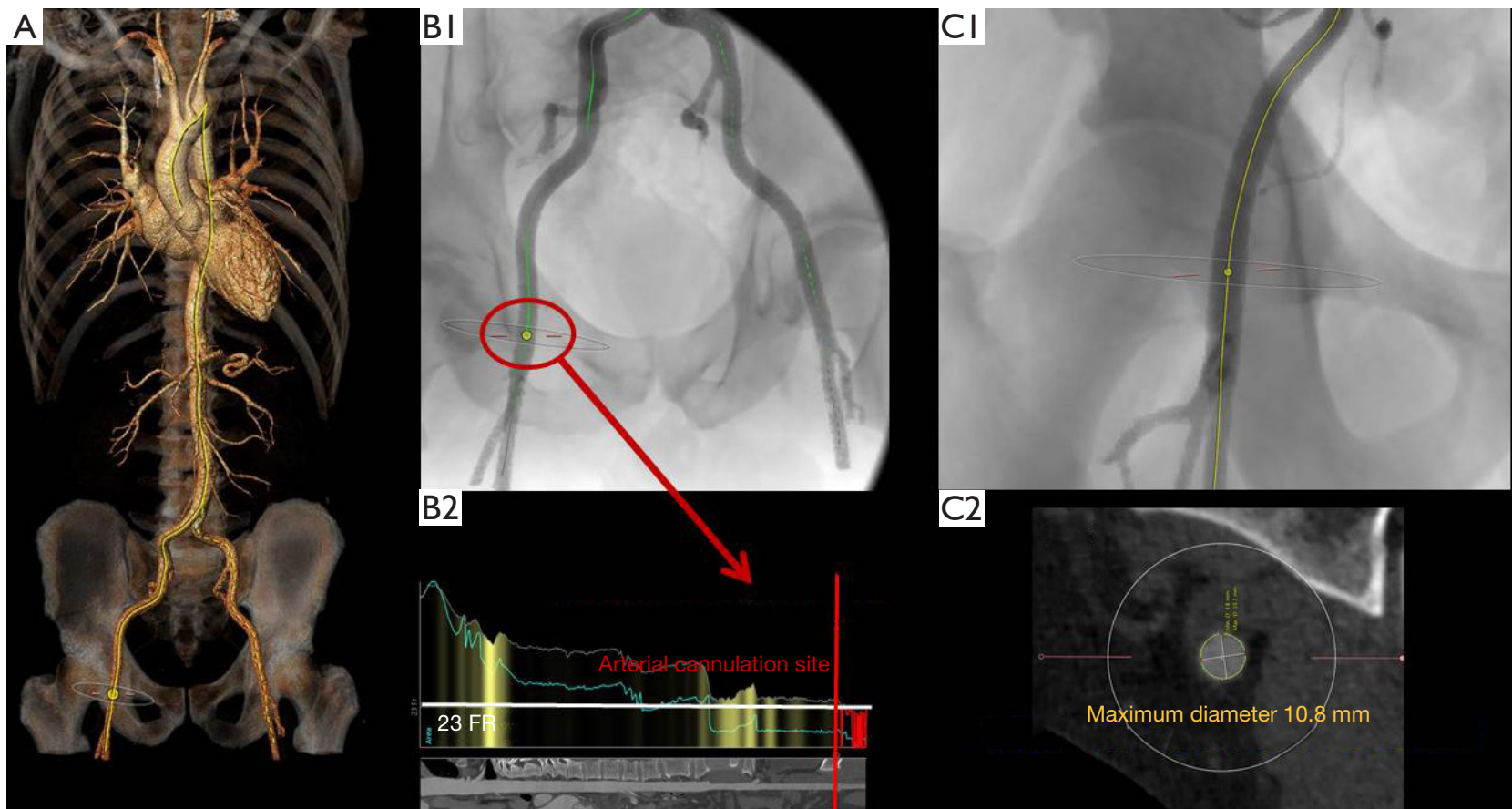

Figure 3 Assessment of the trans-femoral, trans-subclavian or even direct aortic approach to define the optimal cannulation route for MIMVS. (A) 3D reconstruction of a preoperative CTA of the greater vessels. (B1-2) Automated snake-view of the RCFA, the iliac vessels as well as the descending and ascending aorta. At the height of the right femoral head, insertion of a 23 FR arterial cannula into the RCFA will not be totally occlusive. (C1-2) The RCFA was measured to have a maximum diameter of $10.8 \mathrm{~mm}$.

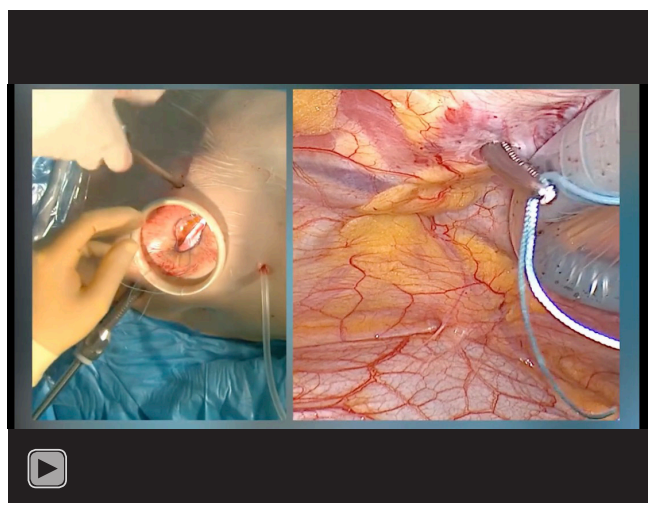

Video 1 A declarative video describing the trans-thoracic external aortic cross-clamping technique in the setting of truly minimally invasive surgical mitral valve repair. Additionally, this video shows the pre-operative planning of the procedure, the complete minimally invasive set-up, and elaborates on the pearls and pitfalls that are associated with this operation.
MVRepair in male patients entails a very limited $3 \mathrm{~cm}$ convex incision that straddles the right areolar border (12).

\section{Cannulation \& cardiopulmonary bypass}

There are four goals of cannulation and perfusion; to provide complete drainage to the heart, to provide adequate systemic perfusion, to minimize risks of malperfusion or vascular injury and to minimize extra time and costs (13). CTA is indispensable in choosing arterial access and planning of the fully percutaneous approach. The larger, less diseased common femoral artery is generally used for arterial perfusion for minimally invasive cardiac surgery. The size and suitability of the femoral artery can be foreseen by CTA measurement. In situations where the vessel is not diseased but simply very small, the addition of a distal perfusion cannula can be considered. However, this is rarely 
used by our group as CPB times tend to be relatively short. Depending on the size of the common femoral artery and the required predicted flow, arterial cannulas ranging from 16 to $20 \mathrm{FR}$ are typically used for arterial perfusion. If the size of the arterial cannula is rather small, a higher arterial line pressure should be anticipated. It is critical to monitor correct guidewire placement by TEE before inserting the cannulas in the Seldinger technique. Right or left axillary artery cannulation can be used if the femoral system is not suitable for arterial inflow or to avoid retrograde flow in case of a "dirty" aorta to avert a stroke. MIMVR becomes unquestionably more challenging without adequate venous drainage as visualization can be impaired and myocardial protection can also become compromised. Under TEE guidance, a multistage or a stented partially covered femoral venous catheter is typically placed from the right femoral vein and finally positioned in the superior vena cava (SVC). The right femoral vein has a more favorable relationship with the iliac artery and is therefore usually preferred over the left one. Venous drainage can be aided by placing a second venous line (15FR to $18 \mathrm{FR}$ ) into the SVC. This can be done by using a low right jugular approach and is either done by the anesthesiology team or by the surgeon, using a small previously placed sheath. In this case, the femoral venous cannula would only be advanced to the IVC right atrial junction. Adding an SVC cannula is especially useful in the setting of tricuspid or ASD procedures. An alternative technique would be to use a single femoral dualstage cannula which does allow for a complete bypass. At the author's institution the standard approach is to only use one venous cannula inserted via the right common femoral vein. Furthermore, every patient receives cranial nearinfrared spectroscopy (NIRS) monitoring intraoperatively heralding potential problems regarding upper-body venous drainage. In case NIRS is dropping uni- or bilaterally in combination with relatively high central venous pressure values, the position of the cannula needs to be corrected. The usual scenario entails a dislodgement of the cannula tip from the SVC into the right atrial appendage after mitral valve exposure. A very easy bail-out manoeuvre in case of inadequate overall drainage is to place a second small (19F) femoral venous cannula through the contralateral femoral vein. With regard to $\mathrm{CPB}$, body temperature is maintained around $34^{\circ} \mathrm{C}, \mathrm{DO}_{2}$ guided-perfusion is utilized and vacuumassisted (20-60 $\mathrm{mmHg}$ ) venous drainage is used throughout the whole procedure. At the author's institution, $\mathrm{DO}_{2}$-guided perfusion is mainly used to safely lower the arterial pumpflow to optimize drainage. Typical flow rates are $80 \%$ of the pre-calculated flow based on an index of $2.4 \mathrm{~L} / \mathrm{min} / \mathrm{m}^{2}$.

\section{Direct external aortic cross-clamping with a trans-thoracic clamp \& cardiac protection}

Most MIMVR surgeons start by performing trans-thoracic external aortic cross-clamping. This technique is familiar and enables the direct clamping of the aorta in the same way as one would do in a sternotomy. The technique is inexpensive as clamps are reusable. It works like a 'lobster pincer' since only one of the two branches is actuated by the handle while the other is straight (14). The trans-thoracic cross-clamp is placed through an entry site in the chest wall, anterior to the SVC. To avoid potential obstruction of the upper-body venous drainage from the cross-clamp, bicaval cannulation or correct positioning of a single venous femoral cannula above the area where the crossclamp crosses to the SVC should be attained. The transthoracic external aortic cross-clamp can be inserted via a small stab wound through the second or third ICS at the right midaxillary line and positioned near the ascending aorta. While clamping, the surgeon needs to make sure not to harm the LA appendage or the left and right pulmonary artery. Apart from that, the antegrade cardioplegia puncture hole is of primal importance. Some surgeons dissect the plane using electrocautery between the ascending aorta and the right pulmonary artery and then place their transthoracic clamp more cranially in order to gain a greater landing zone for the antegrade cardioplegia catheter. Also, to have their antegrade cardioplegia catheter at the fatty band region on the right lateral side of the ascending aorta because this is where the stronger and healthier epiaortic tissue is. Moreover, if the surgeon is choosing the option of putting his cross-clamp caudally from the right pulmonary artery without dissecting the tissue between the aorta and the pulmonary artery, he will have to perform blunt dissection with the suction and make the transverse sinus free (Figure 4A). On the other hand, a second clamp can be utilized in addition to the primary trans-thoracic cross-clamp, which will pull the ascending aorta through the lateral mini-thoracotomy caudally after reducing pump flow. Then, closing the primary trans-thoracic cross-clamp which is placed through the second or third ICS at the right midaxillary line (Figure 4B). This results in a larger epiaortic surface to place the antegrade cardioplegia catheter (Figure $4 C$ ). Next, the surgeon punctures above the aortic fatty band without placing any aortic root suture thus saving another stab wound into the chest to externalize any prolene 


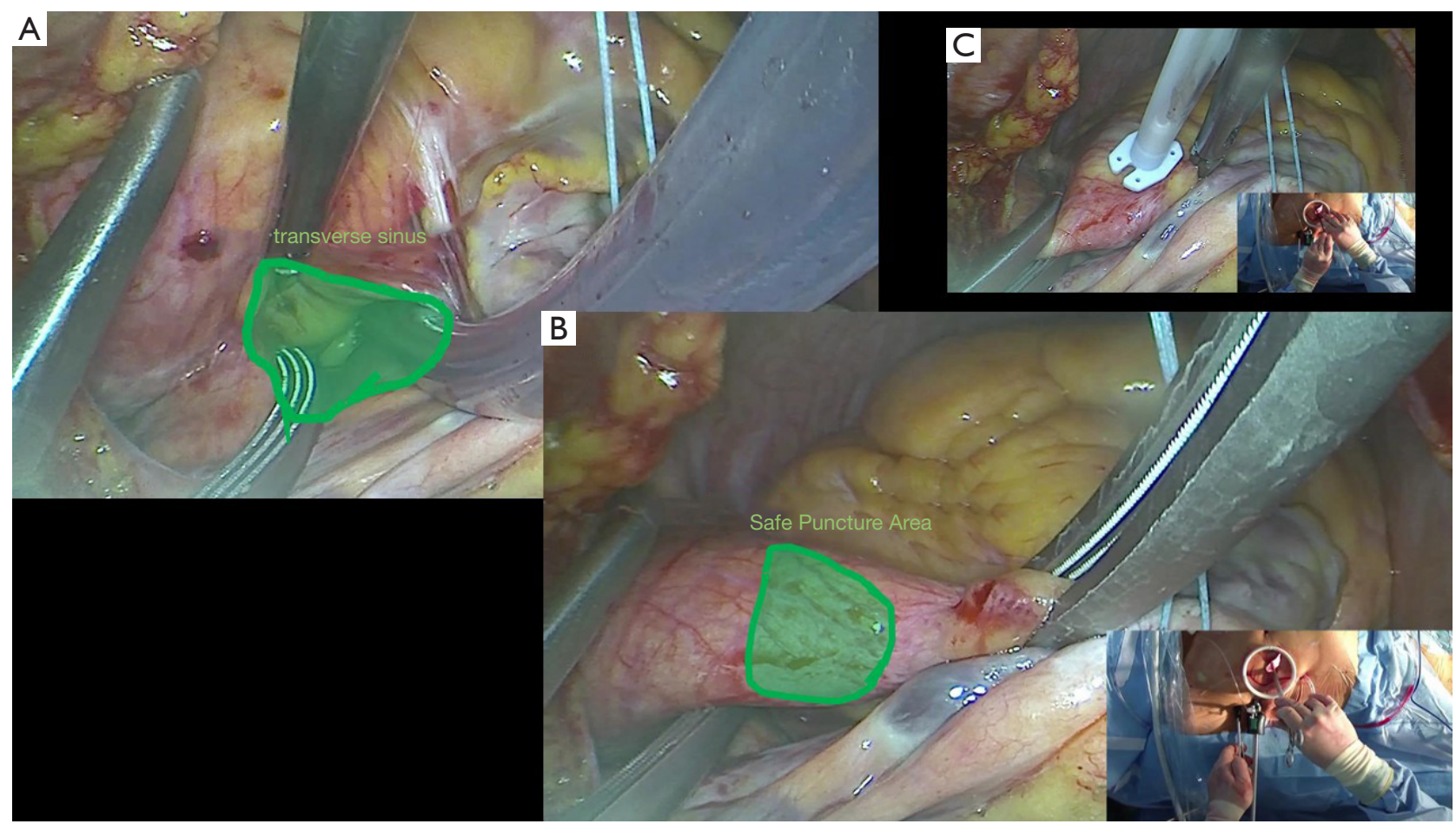

Figure 4 Introduction and positioning of the trans-thoracic external aortic cross-clamp. (A) Clearing the transverse sinus and placing of the trans-thoracic clamp; (B) clamping of the ascending aorta while pulling the aorta caudally with a second clamp in order to gain greater epi-

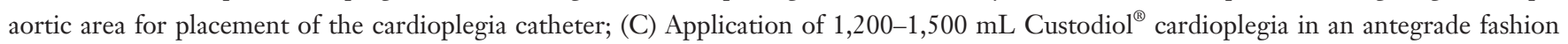
with line-pressures of approximately $150 \mathrm{mmHg}$.

sutures (Figure $4 C$ ). Afterwards, Custodiol ${ }^{\circledR}$ cardioplegia is delivered directly into the aortic root with a pressure of $150 \mathrm{mmHg}$ on the line, resulting in a rate of approximately $300 \mathrm{~mL}$ per minute (Figure 4C). In general, between 1,200$1,500 \mathrm{~mL}$ of Custodiol $^{\circledR}$ is given as a single shot depending on preoperative cardiac function and left ventricular (LV) mass. Subsequently, when cardioplegia is administered, the antegrade cardioplegia catheter is pulled out of the surgical field with the puncture hole left open.

\section{High definition 3D endoscopic visualization}

Once the thorax has been entered, a high definition $30^{\circ}$ thoracoscope is placed into the chest via a $10 \mathrm{~mm}$ port at the same ICS at the right anterior axillary line but below the mini-thoracotomy for high definition fully endoscopic 3D MIMVS. The thoracoscope not only provides an additional view from which to perform subsequent work but also brightly illuminates the entire chest. Throughout the procedure, the surgical field is flooded with carbon dioxide $\left(\mathrm{CO}_{2}\right)$ through the camera port.

\section{Intrathoracic and mitral valve exposure (Figure 5)}

In case the right hemi-diaphragm is found to be too high cranially into the thoracic cage after mini-thoracotomy, often seen in obese patients, the right hemi-diaphragm can be retracted caudally and to the right with a superficial suture placed in the tendinous dome. This is then brought out by a suture hook through a stab incision in the right sixth or seventh intercostal space. The pericardium is opened $4-5 \mathrm{~cm}$ anteriorly and parallel to the phrenic nerve from the distal ascending aorta to the diaphragm.

\section{Surgical mitral valve repair (Figure 6)}

The MV is accessed through an incision into the Waterston's groove and a LA retractor is used to expose the MV. MV repair for degenerative MV disease is most commonly performed utilising the Gore-Tex neochordae using the "Loop technique", the details of which have been described by Ulrich Otto von Oppell and Friedrich W. Mohr (15). The fundamental aspects of this technique (16) 

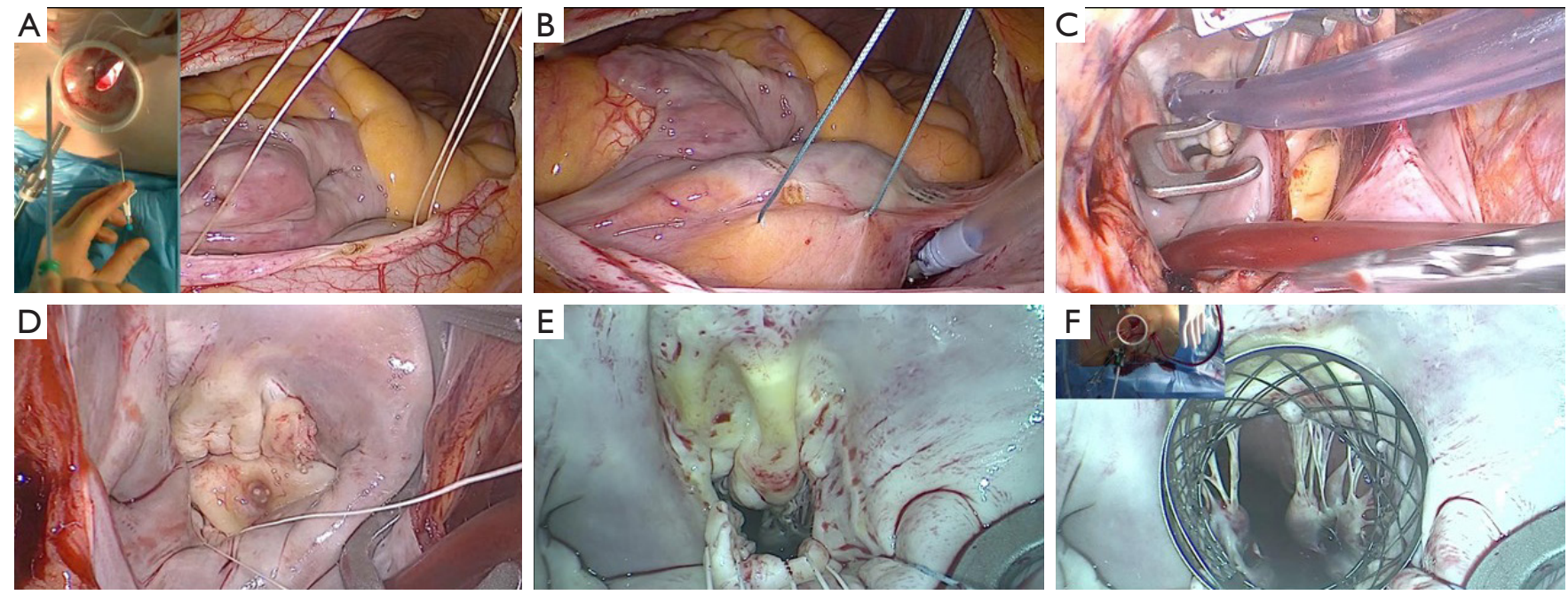

Figure 5 Intrathoracic visualization and MV exposure. (A) The opened pericardium is pulled laterally with two 2-0 vicryl sutures without too much tension to prevent phrenic nerve paresis. (B) Placement of a suture in the fatty region covering the interatrial (Waterston) groove. During trans-thoracic external aortic cross-clamping this suture lays loosely reposed in the thorax so as not to impede the surgeon's visibility whilst clamping the aorta. After administration of cardioplegia and shortly before performing the left atriotomy, this suture gets pulled up to expose both right pulmonary veins. (C) Arrangement of the LA retractor (through the fifth ICS parasternally) and the venting catheter. (D) Complete exposition of the MV with the LA retractor and the venting catheter in optimal position. (E) To enhance the visibility of the subvalvular apparatus Gore-Tex sutures can be placed through the free margin of the prolapsing segment of the posterior leaflet (PML). This maneuver heaves the PML posteriorly thereby opening up the orifice of the MV. (F) The surgeon can work with a flexible nitinol band (Superflex Soft Tissue Retractor by Fehling Instruments, Germany) to open up the MV and precisely inspect the subvalvular apparatus and place pre-measured loops in case of leaflet repair.

are the assessment of the optimal length and precise fixation of neochordae to the papillary muscles and the free edge of the mitral leaflets on the level of the mitral annulus. An annuloplasty ring is implanted to support the repair and the geometric dynamics of the mitral valve. Sutures can be either tied manually using a knot-pusher or with the help of the CorKnot device (LSI). MV competency is restored in patients with Barlow's disease, utilising different techniques from leaflet resection to neochordae to Alfieri's edgeto-edge repair $(6,17)$. Functional mitral regurgitation is corrected utilising an undersized annuloplasty ring alone or with the addition of subvalvular repair techniques (18).

\section{De-airing \& closure (Figure 7)}

After completing the mitral procedure and flooding the $\mathrm{LV}$ with $\mathrm{CO}_{2}$, the vent can either be placed in the $\mathrm{LV}$ (enhances de-airing) or in the LA only (eases LA closure). Antegrade de-airing through the cardioplegia puncture hole (by gentle cardiac compressions with a sponge stick) as well as retrograde de-airing via the left atriotomy is then completed using modest inflation of the lungs in addition to relying on the $\mathrm{CO}_{2}$ to flood the operative field.

The cardioplegia hole is secured by one felt supported 4-0 U-stich which can be tied manually or by using a CorKnot. Finally, the external trans-thoracic cross-clamp is released under non-pressurized conditions. Epicardial pacing wires should be placed while the heart is still decompressed on CPB. Following this, separation from CPB, decannulation, TEE examination of adequacy of mitral repair, and reversal of anticoagulation are all conducted in a standard fashion.

\section{Clinical results \& discussion}

Endo-aortic balloon occlusion is the alternative to the transthoracic external aortic cross-clamp (7,19-25). The resulting absence of a cardioplegia puncture hole in the ascending aorta is a distinct advantage of the endo-aortic balloon occlusion technique. This eradicates the need to suture the aorta and the risk of bleeding from the puncture site (19). It is also useful for re-do procedures where external cross-clamping is cumbersome due to adhesions $(19,26)$. 

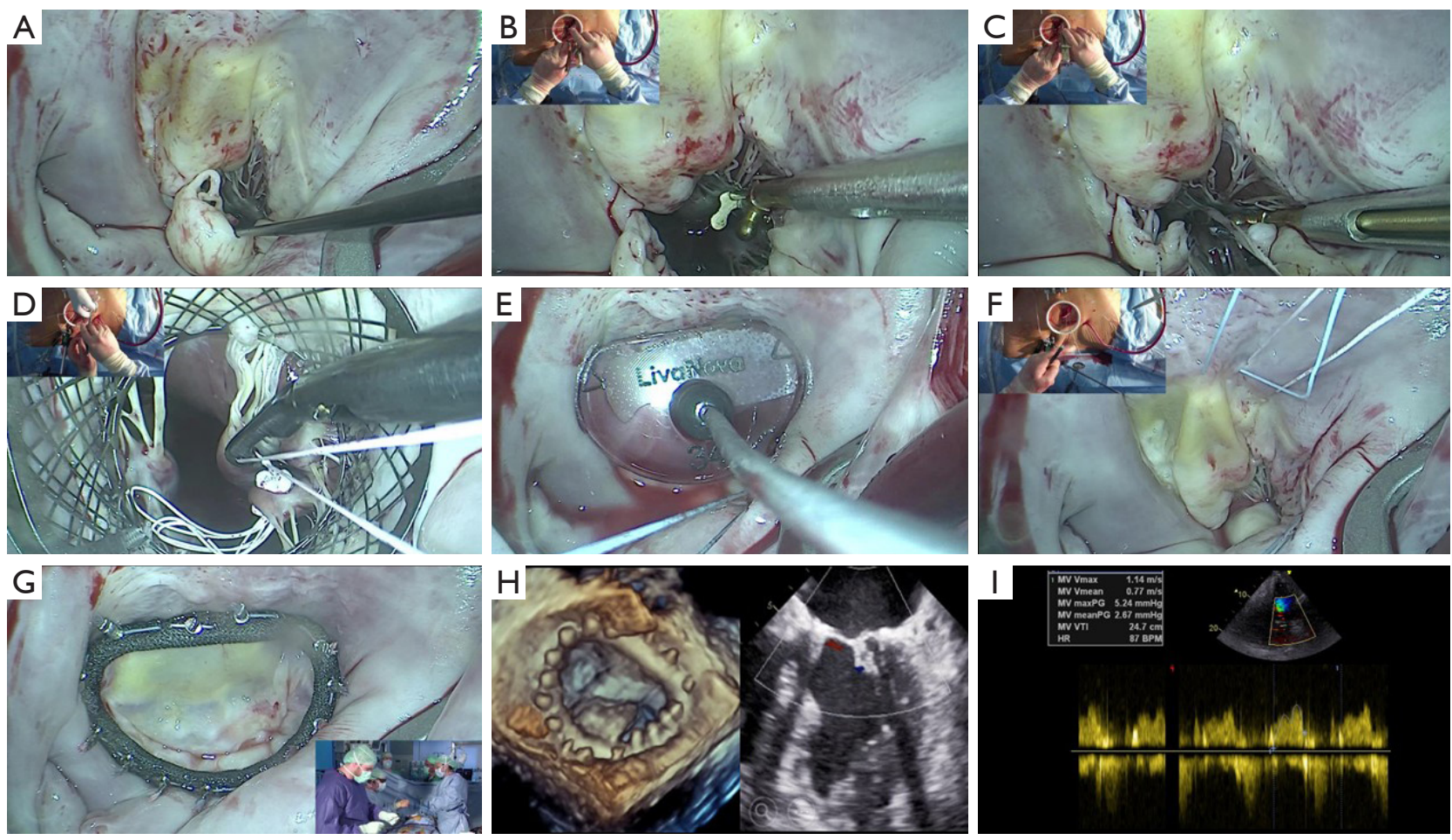

Figure 6 Surgical MV repair in a patient with P2-prolapse making use of one set of $14 \mathrm{~mm}$ pre-measured loops and an annuloplasty ring. (A) Inspection of the MV and identification of prolapsing segments and clefts; (B) measurement of the appropriate loop length using a long-shafted endoscopic ruler (14 mm pre-measured loops in this case); (C) simulating the effect of the chosen loop length by flipping the excess tissue into the ventricle (D) implantation of $14 \mathrm{~mm}$ pre-measured loops; (E) annuloplasty ring sizing, based on the anterior mitral leaflet length and CC distance; (F) placement of the annuloplasty ring sutures. (G) the repaired MV under pressurized conditions to control residual leakage and geometry of the closure line; (H) TEE 3D reconstruction of the repaired MV without residual insufficiency; (I) 2.67 $\mathrm{mmHg}$ mean pressure gradient over the repaired $\mathrm{MV}$.

The disadvantages of the endo-aortic balloon occlusion technique include having to place the catheter through the arterial cannula thereby reducing the effective size of the arterial cannula (19). A second arterial cannula can be placed if the result is high line pressures or inadequate flow (19). It has also been observed that the distal balloon can migrate distally resulting in innominate artery occlusion (19). Distal balloon migration can, however, be easily recognized by monitoring bilateral radial artery pressures. The potential for migrating can also be tempered by correct positioning and pulling the slack out of the catheter while inflating the balloon. Concerns were raised in the early days regarding the endoaortic balloon's association with aortic dissection (19). But this was at a time before CT angiography was routinely implemented to assess the general risk of retrograde perfusion in certain anatomies and before the importance of training in basic wire skills were accepted (19). Proximal migration has also been observed and this has the potential to obscure the operative field, especially the left fibrous trigone (19). It may also become non-occlusive to the sinus of Valsalva, resulting in perfusion of the coronary ostia (19). Other disadvantages are possible balloon puncture or rupture during the procedure, the need for bilateral arterial lines to monitor placement and the cost of the catheter itself (19). The data comparing trans-thoracic external aortic cross-clamping with endoaortic balloon occlusion are limited and solely retrospective, highlighting the fact that there is no significant difference in the safety profiles of the two techniques (7-9,19,27-32). Both techniques have a considerably lower stroke rate than fibrillatory arrest but there is no difference in retrograde aortic dissection, bleeding, or adequacy of myocardial protection $(13,19,23,33-35)$. In their retrospective study, 

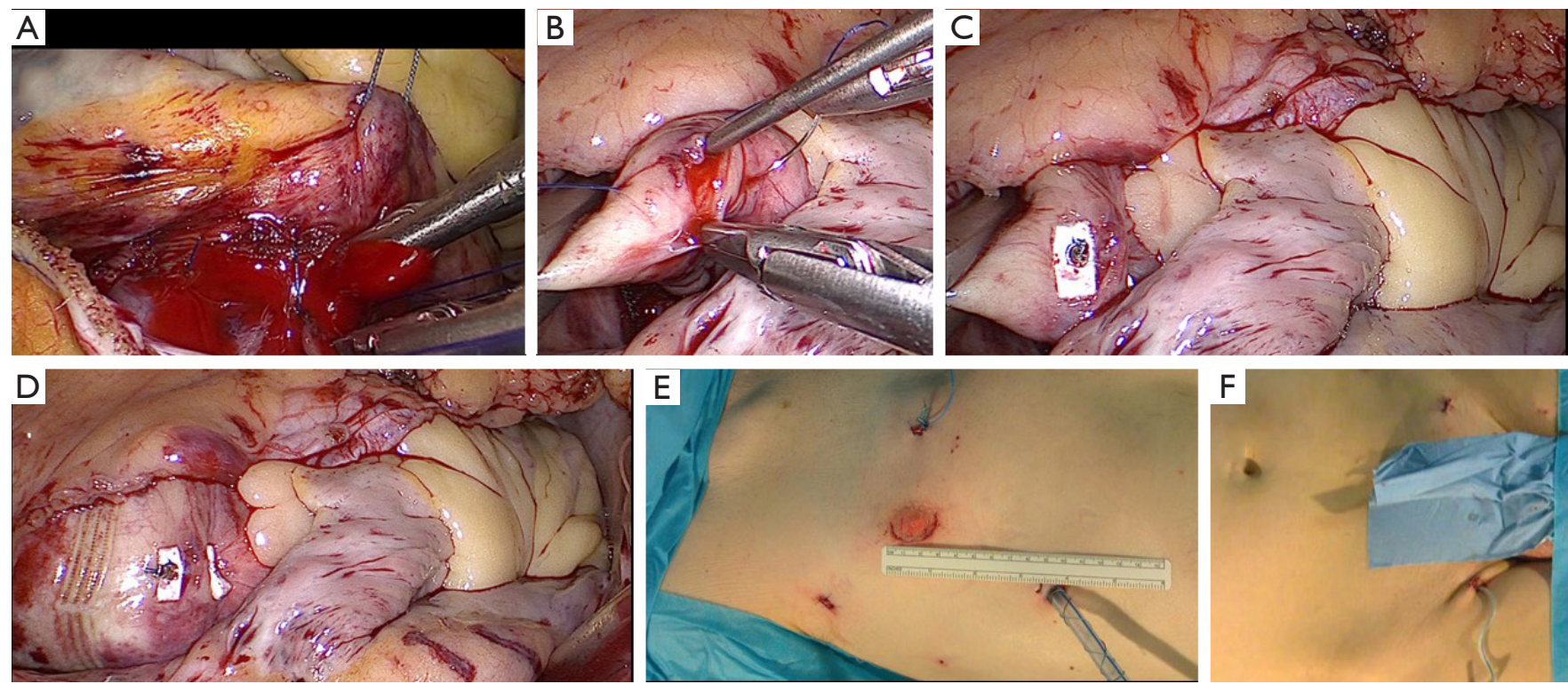

Figure 7 De-airing of the LA and LV \& closure of the left atriotomy and the right anterolateral periareolar incision. (A) Retrograde deairing via the left atriotomy with modest inflation of the lungs in addition to relying on the $\mathrm{CO}_{2}$ for flooding of the operative field. (B) Suturing the cardioplegia hole with a 4-0 prolene pledged suture. The suture is tied using the Cor-Knot ${ }^{\circledR}$ device. (C) Closure of the cardioplegia puncture hole under depressurized conditions with the trans-thoracic external aortic cross-clamp still in place. (D) Removal of the trans-thoracic external aortic cross-clamp. (E,F) Final result after de-cannulation and closure of the periareolar incision.

Murzi et al. (36) concluded that MIMVS with transthoracic external aortic cross-clamping is a safe and effective procedure. The use of retrograde perfusion in older patients with atherosclerotic burden is associated with an increased risk of postoperative stroke (36). Yet, retrograde perfusion (femoral cannulation) remains a viable option for younger patients without vascular comorbidities (36). If a femoral perfusion technique is chosen in older patients, preoperative evaluation of the aorta and distal vasculature (CT-scan) is indicated to demonstrate that the individual patient is not exposed to an increased neurological risk (36).

Moreover, according to the ISMICS consensus paper of 2011 (37), which compared MIMVS to open mitral valve surgery, MIMVS required longer operative times (19). It also stated that MIMVS had higher incidences of stroke, aortic dissection, phrenic nerve palsy and groin infection rates $(19,37)$. In contrast, MIMVS was associated with a reduction in hospital and intensive care unit stay, in transfusion rate and in the occurrence of postoperative atrial fibrillation and ventilation time $(19,37,38)$. Additionally, Schneider et al. (39) showed that transcranial Doppler was useful to detect cerebral microemboli in MIMVS and conventional MV operations. Nonetheless, they found no increased risk of cerebral micro-embolism during the minimally invasive method com- pared with the conventional technique (39). In addition, Casselman et al. (28) proved within a retrospective European multicenter study that stroke rate and observed mortality compared favourably with the existing literature on MV surgery irrespective of the approach. The ISMICS statement reports that these observations were not based on randomized prospective studies but almost solely on observational studies $(19,37)$. These issues have yet to be definitively addressed and it is true, even today, that no randomized prospective studies have been conducted $(19,37)$. Even the ISMICS statement was founded on literature published before March 2010 and, as they admitted, included only "retrospective studies with important differences in baseline patient characteristics" $(19,37)$. But a few observations can be made. Before 2010 most MIMVR surgeons did not use CTA which might explain the higher incidence of stroke and aortic dissection $(19,37)$. The authors went into great detail concerning the use of CTA in preoperative planning in order to avoid these complications (19). The ISMICS consensus statement did not show an increased mortality when compared to open MV repair (37). In regard to groin complications (lymphocele, arterial complications, infection), the incidence have become rare (19) and some groups have 
started to utilize percutaneous cannulation techniques with good results (40). It is important to note that most MIMVS surgeons today are well aware of the chance of phrenic nerve palsy and have developed techniques to avoid its occurrence (19).

Onnasch et al. (41) described a series of 39 patients who underwent redo MV surgery via the MIMVS approach with no adhesions in $84 \%$ of the patients. However, in their study, the trans-thoracic direct external aortic crossclamping was avoided, and hence, there was no need for dissection of the ascending aorta and the pulmonary artery trunk (41). But they showed that the right anterolateral minithoracotomy technique has become a standard approach for redo MV procedures and that it could be performed safely with a reduction in possible injury to cardiac structures such as previous coronary artery bypass conduits (venous and internal mammary artery grafts) (41). In their retrospective analysis, Mazine et al. (34) reported the use of the trans-thoracic external aortic cross-clamping technique in three redo cases, yet the authors did not conduct a comparison between the two established crossclamping techniques within this subgroup. Still, the desired approach at the author's institution in the case of a redo MV procedure via a lateral mini-thoracotomy would be application of the endo-aortic balloon occlusion technique or on a fibrillating heart. Having said that, this does not mean that in certain clinical situations and selected patients the trans-thoracic external aortic cross-clamp could not be a good option.

To summarize, all MIMVS team members must possess a deep understanding of the procedure, its potential pitfalls and complications (19). For a successful MIMVS program, it is essential that there is a selection of ideal patients and a well-developed preoperative plan involving the operating room team (19). This team is made up of anesthesiologists, perfusionists and surgeons, all of whom are comfortable with MV surgery including conventional techniques for repair (19).

\section{Acknowledgments}

Funding: None.

\section{Footnote}

Provenance and Peer Review: This article was commissioned by the Guest Editors (Filip Casselman and Johan van der Merwe) for the series "Aortic and Mitral Valve Innovative
Surgery" published in fournal of Visualized Surgery. The article has undergone external peer review.

Conflicts of Interest: All authors have completed the ICMJE uniform disclosure form (available at https://jovs. amegroups.com/article/view/10.21037/jovs-2019-17/coif). The series "Aortic and Mitral Valve Innovative Surgery" was commissioned by the editorial office without any sponsorship or funding. VF reports grants from Abbott $\mathrm{GmbH} \& \mathrm{Co}, \mathrm{KG}$, grants from Medtronic $\mathrm{GmbH}$, grants from Boston Scientific, grants from Edwards Lifesciences, grants from JOTEC GmbH, other from Biotronik Se \& Co, other from Berlin Heart, other from Novartis Pharma $\mathrm{GmbH}$, outside the submitted work. JK reports personal fees from Edwards, personal fees from LSI, outside the submitted work. The authors have no other conflicts of interest to declare.

Etbical Statement: The authors are accountable for all aspects of the work in ensuring that questions related to the accuracy or integrity of any part of the work are appropriately investigated and resolved. All procedures performed in this study were in accordance with the Helsinki Declaration (as revised in 2013). The manuscript is waived from patient informed consent according to the ethics committee or institutional review board.

Open Access Statement: This is an Open Access article distributed in accordance with the Creative Commons Attribution-NonCommercial-NoDerivs 4.0 International License (CC BY-NC-ND 4.0), which permits the noncommercial replication and distribution of the article with the strict proviso that no changes or edits are made and the original work is properly cited (including links to both the formal publication through the relevant DOI and the license). See: https://creativecommons.org/licenses/by-nc-nd/4.0/.

\section{References}

1. Modi P, Hassan A, Chitwood WR Jr. Minimally invasive mitral valve surgery : a systematic review and metaanalysis. Eur J Cardiothorac Surg 2008;34:943-52.

2. Schmitto JD, Mokashi SA, Cohn LH. Minimally-Invasive Valve Surgery. J Am Coll Cardiol 2010;56:455-62.

3. Van Praet KM, Stamm C, Starck CT, et al. An overview of surgical treatment modalities and emerging transcatheter interventions in the management of tricuspid valve regurgitation. Expert Rev Cardiovasc Ther 2018;16:75-89. 
4. Mishra YK, Malhotra R, Mehta Y, et al. Minimally Invasive Mitral Valve Surgery Through Right Anterolateral Minithoracotomy. Ann Thorac Surg 1999;68:1520-4.

5. Schroeyers P, Wellens F, De Geest R, et al. Minimally Invasive Video-Assisted Mitral Valve Repair: Short and Mid-Term Results. J Heart Valve Dis 2001;10:579-83.

6. Van Praet KM, Stamm C, Sündermann SH, et al. Minimally invasive surgical mitral valve repair: State of the art review. Interv Cardiol 2018;13:14-9.

7. Barbero C, Krakor R, Bentala M, et al. Comparison of Endoaortic and Transthoracic Aortic Clamping in Less-Invasive Mitral Valve Surgery. Ann Thorac Surg 2018;105:794-8.

8. Aybek T, Dogan S, Westphal K, Mortiz A. The MicroMitraI Operation Comparing the Port-Access Technique and the Transthoracic Clamp Technique. J Card Surg 2000;15:76-81.

9. Reichenspurner H, Detter C, Deuse T, et al. Video and Robotic-Assisted Minimally Invasive Mitral Valve Surgery : A Comparison of the Port- Access and Transthoracic Clamp Techniques. Ann Thorac Surg 2005;79:485-90.

10. Rival PM, Moore THM, McAleenan A, et al. Transthoracic clamp versus endoaortic balloon occlusion in minimally invasive mitral valve surgery: A systematic review and meta-analysis. Eur J Cardiothorac Surg 2019;56:643-53.

11. Chan EY, Lumbao DM, Iribarne A, et al. Evolution of Cannulation Techniques for Minimally Invasive Cardiac Surgery. Innovations (Phila) 2012;7:9-14.

12. Van Praet KM, Stamm C, Sündermann SH, et al. Minimally Invasive Cardiac Surgery: Removal of an Interatrial Intraseptal Bronchogenic Cyst Through a Periareolar Approach. Innovations (Phila) 2018;13:230-2.

13. Ad N, Holmes SD, Shuman DJ, Pritchard G, Massimiano PS. Minimally invasive mitral valve surgery without aortic cross-clamping and with femoral cannulation is not associated with increased risk of stroke compared with traditional mitral valve surgery: A propensity score-matched analysis. Eur J Cardiothorac Surg 2015;48:868-72.

14. Maselli D, Pizio R, Borelli G, et al. Endovascular balloon versus transthoracic aortic clamping for minimally invasive mitral valve surgery : impact on cerebral microemboli. Interact Cardiovasc Thorac Surg 2006;5:183-6.

15. von Oppell UO, Mohr FW. Chordal Replacement for Both Minimally Invasive and Conventional Mitral Valve Surgery Using Premeasured Gore-Tex Loops. Ann Thorac Surg 2000;70:2166-8.
16. Davierwala PM, Seeburger J, Pfannmueller B, et al. Minimally invasive mitral valve surgery : "The Leipzig experience”. Ann Cardiothorac Surg 2013;2:744-50.

17. Coutinho GF, Antunes MJ. Mitral valve repair for degenerative mitral valve disease: Surgical approach, patient selection and long-term outcomes. Heart 2017;103:1663-9.

18. Girdauskas E, Pausch J, Harmel E, et al. Minimally invasive mitral valve repair for functional mitral regurgitation. Eur J Cardiothorac Surg 2019;55:117-25.

19. Ailawadi G, Agnihotri AK, Mehall JR, et al. Minimally Invasive Mitral Valve Surgery I - Patient Selection, Evaluation, and Planning. Innovations (Phila) 2016;11:243-50.

20. Bentala M, Heuts S, Vos R, et al. Comparing the endoaortic balloon and the external aortic clamp in minimally invasive mitral valve surgery. Interact Cardiovasc Thorac Surg 2015;21:359-65.

21. Malvindi PG, Margari V, Mastro F, et al. External aortic cross-clamping and endoaortic balloon occlusion in minimally invasive mitral valve surgery. Ann Cardiothorac Surg 2018;7:748-54.

22. Barbero C, Ricci D, Qarra S El, Marchetto G. Aortic cannulation system for minimally invasive mitral valve surgery. J Thorac Cardiovasc Surg 2015;149:1669-72.

23. Umakanthan R, Leacche M, Petracek MR, et al. Safety of Minimally Invasive Mitral Valve Surgery Without Aortic Cross-Clamp. Ann Thorac Surg 2008;85:1544-9.

24. Barbero C, Marchetto G, Ricci D, et al. Minimal access surgery for mitral valve endocarditis. Interact Cardiovasc Thorac Surg 2017;25:241-5.

25. Rinaldi M, Krakor R, Bentala M et al. Endoaortic balloon clamping and transthoracic aortic clamping are both safe and effective in minimally invasive mitral valve surgery. Interact Cardiovasc Thorac Surg 2014;19:S69.

26. van der Merwe J, Casselman F, Stockman B, et al. Late redo-port access surgery after port access surgery. Interact Cardiovasc Thorac Surg 2016;22:13-8.

27. Liebold A, Skrabal C, Emini R, et al. Improved outcome of minimally invasive mitral surgery with IntraClude aortic balloon occlusion compared to transthoracic clamping. Pap Present 14 ISMICS; May 28-31, 2014, Boston, MA. Available online: http//epostersonline.s3.amazonaws.com/ ismics2014/ismics201409c0088NORMAL.:2.

28. Casselman F, Aramendi J, Bentala M, et al. Endoaortic Clamping Does Not Increase the Risk of Stroke in Minimal Access Mitral Valve Surgery : A Multicenter Experience. Ann Thorac Surg 2015;100:1334-9. 
29. Atluri P, Goldstone AB, Fox J, et al. Port Access Cardiac Operations Can Be Safely Performed With Either Endoaortic Balloon or Chitwood Clamp. Ann Thorac Surg 2014;98:1579-83.

30. Modi P, Rodriguez E, Clark W, et al. Minimally invasive video-assisted mitral valve surgery : A 12-year, 2-center experience in 1178 patients. J Thorac Cardiovasc Surg 2009;137:1481-7.

31. Krapf C, Wohlrab P, Häußinger S, et al. Remote access perfusion for minimally invasive cardiac surgery : to clamp or to in fl ate? †. Eur J Cardiothorac Surg 2013;44:898-904.

32. Kempfert J, Meyer A, Cetinkaya A, et al. Propensity matched comparison of endoclamping versus direct aortic clamping for minimally invasive mitral valve surgery. Pap Present 15 ISMICS; June 3-6, 2015, Berlin, Germany. Available online: http//meetings.ismics.org/abstracts/2015/ P99.cgi, Accessed January 31, 2016.

33. Gammie JS, Zhao Y, Peterson ED, et al. Less-Invasive Mitral Valve Operations : Trends and Outcomes From The Society of Thoracic Surgeons Adult Cardiac Surgery Database. Ann Thorac Surg 2010;90:1401-8.

34. Mazine A, Pellerin M, Dionne P, et al. Minimally Invasive Mitral Valve Surgery : Influence of Aortic Clamping Technique on Early Outcomes. Ann Thorac Surg 2013;96:2116-22.

35. Aybek T, Dogan S, Westphal K, et al. The Micro-

doi: 10.21037/jovs-2019-17

Cite this article as: Van Praet KM, Kofler M, Montagner M, Heck R, Eggert-Doktor D, Stamm C, Unbehaun A, Jacobs S, Falk V, Kempfert J. Minimally invasive mitral valve repair using external clamping_-pearls and pitfalls. J Vis Surg 2020;6:45.
Mitral Operation Comparing the Port-Access Technique and the Transthoracic Clamp Technique. J Card Surg 2000;15:76-81.

36. Murzi M, Cerillo AG, Gasbarri T, et al. Antegrade and retrograde perfusion in minimally invasive mitral valve surgery with transthoracic aortic clamping: A singleinstitution experience with 1632 patients over 12 years. Interact Cardiovasc Thorac Surg 2017;24:363-8.

37. Falk V, Cheng DCH, Diegeler A, et al. Minimally invasive versus open mitral valve surgery: a consensus statement of the International Society of Minimally Invasive Cardiac Surgery. (ISMICS) 2010. Innovations (Phila) 2011;6:66-76.

38. Grossi EA, Galloway AC, Lapietra A, et al. Minimally Invasive Mitral Valve Surgery: A 6-Year Experience With 714 Patients. Ann Thorac Surg 2002;74:660-3.

39. Schneider F, Onnasch JF, Falk V, et al. Cerebral Microemboli During Minimally Invasive and Conventional Mitral Valve Operations. Ann Thorac Surg 2000;70:1094-7.

40. Doenst T, Diab M, Sponholz C, et al. The Opportunities and Limitations of Minimally Invasive Cardiac Surgery. Dtsch Arztebl Int 2017;114:777-84.

41. Onnasch JF, Schneider F, Falk V, et al. Minimally lnvasive Approach for Redo MitraI Valve Surgery : A True Benefit for the Patient. J Card Surg 2002;17:14-9. 